\title{
Correction to: Exploring the missing heritability in subjects with hearing loss, enlarged vestibular aqueducts, and a single or no pathogenic SLC26A4 variant
}

\author{
Jeroen J. Smits ${ }^{1,2,3}$ - Suzanne E. de Bruijn ${ }^{2,3}$ - Cornelis P. Lanting ${ }^{1}\left(\mathbb{D} \cdot\right.$ Jaap Oostrik $^{1,3} \cdot$ Luke O'Gorman $^{2}$. \\ Tuomo Mantere ${ }^{2,4}$. DOOFNL Consortium • Frans P. M. Cremers ${ }^{2,3}$ (D) Susanne Roosing ${ }^{2,3} \cdot$ Helger G. Yntema ${ }^{2}$. \\ Erik de Vrieze $^{1,3} \cdot$ Ronny Derks $^{2} \cdot$ Alexander Hoischen $^{2,5,6,7} \cdot$ Sjoert A. H. Pegge ${ }^{8} \cdot$ Kornelia Neveling $^{2}$. \\ Ronald J. E. Pennings ${ }^{1,3}$ (D) Hannie Kremer ${ }^{1,2,3}$ (i)
}

Published online: 4 October 2021

(c) Springer-Verlag GmbH Germany, part of Springer Nature 2021

\section{Correction to: Human Genetics https://doi.org/10.1007/s00439-021-02336-6}

In the original article published, the affiliation of the 3rd author Cornelis P. Lanting is published incorrectly. The correct affiliation is given below:

1. Hearing and Genes, Department of Otorhinolaryngology, Radboud University Medical Center, Nijmegen, The Netherlands
The original article has been corrected.

Publisher's Note Springer Nature remains neutral with regard to jurisdictional claims in published maps and institutional affiliations.
The original article can be found online at https://doi.org/10.1007/ s00439-021-02336-6.

Hannie Kremer

Hannie.Kremer@radboudumc.nl

1 Hearing and Genes, Department of Otorhinolaryngology, Radboud University Medical Center, Nijmegen, The Netherlands

2 Department of Human Genetics, Radboud University Medical Center, Internal Postal Code 855, P.O. Box 9101, 6500 HB Nijmegen, The Netherlands

3 Donders Institute for Brain, Cognition and Behaviour, Radboud University Medical Center, Nijmegen, The Netherlands

4 Laboratory of Cancer Genetics and Tumor Biology, Cancer and Translational Medicine Research Unit and Biocenter Oulu, University of Oulu, Oulu, Finland
Center for Infectious Diseases (RCI), Department of Internal Medicine, Radboud University Medical Center, Nijmegen, The Netherlands

6 Radboud Institute for Molecular Life Sciences, Radboud University Medical Center, Nijmegen, The Netherlands

7 Radboud Expertise Center for Immunodeficiency and Autoinflammation and Center for Infectious Disease (RCI), Radboud University Medical Center, Nijmegen, The Netherlands

8 Department of Radiology and Nuclear Medicine, Radboud University Medical Center, Nijmegen, The Netherlands 